\title{
A randomised, double-blind, placebo-controlled trial of tropisetron in patients with schizophrenia
}

\author{
Akihiro Shiina', Yukihiko Shirayama², Tomihisa Niitsu³, Tasuku Hashimoto33, Taisuke Yoshida³, Tadashi Hasegawa', \\ Tadashi Haraguchi3 ${ }^{3}$, Nobuhisa Kanahara33, Tetsuya Shiraishi³, Mihisa Fujisaki1', Goro Fukami' , Michiko Nakazato', \\ Masaomi lyo 1,3 and Kenji Hashimoto*4
}

\begin{abstract}
Background: Cognitive deficits in schizophrenia are associated with psychosocial deficits that are primarily responsible for the poor long-term outcome of this disease. Auditory sensory gating P50 deficits are correlated with neuropsychological deficits in attention, one of the principal cognitive disturbances in schizophrenia. Our studies suggest that the a7 nicotinic acetylcholine receptor ( $\mathrm{a} 7 \mathrm{nAChR}$ ) agonist tropisetron might be a potential therapeutic drug for cognitive deficits in schizophrenia. Therefore, it is of particular interest to investigate the effects of tropisetron on the cognitive deficits in patients with schizophrenia.

Methods: A randomised, placebo-controlled trial of tropisetron in patients with schizophrenia was performed. A total of 40 patients with chronic schizophrenia who had taken risperidone (2 to $6 \mathrm{mg} / \mathrm{day}$ ) were enrolled. Subjects were randomly assigned to a fixed titration of tropisetron $(n=20,10 \mathrm{mg} /$ day) or placebo $(n=20)$ in an 8-week double-blind trial. Auditory sensory gating P50 deficits and Quality of Life Scale (QLS), Cambridge Neuropsychological Test Automated Battery (CANTAB), and Positive and Negative Syndrome Scale (PANSS) scores were measured.

Results: In all, 33 patients completed the trial. Tropisetron was well tolerated. Administration of tropisetron, but not placebo, significantly improved auditory sensory gating P50 deficits in non-smoking patients with schizophrenia. The score on the rapid visual information processing (sustained visual attention) task of CANTAB was significantly improved by tropisetron treatment. Total and subscale scores of PANSS were not changed by this trial. QLS scores in the all patients, but not non-smoking patients, were significantly improved by tropisetron trial.
\end{abstract}

Conclusions: This first randomised, double-blind, placebo-controlled trial supports the safety and efficacy of adjunctive tropisetron for treatment of cognitive deficits in schizophrenia.

\section{Background}

Cognitive deficits in schizophrenia are frequently severe and strongly correlated with decreased functional outcome and quality of life (QOL) [1-5]. Atypical antipsychotics have been shown to provide an improvement in several domains of cognitive function, especially working memory, executive function, and attention [2,6]. However, many patients, even if they are medicated with atypical antipsychotics, fail to recover from cognitive deficits, resulting in a failure of their reintegration into society.

* Correspondence: hashimoto@faculty.chiba-u.jp

${ }^{4}$ Division of Clinical Neuroscience, Chiba University Center for Forensic Mental Health, Chiba, Japan

Full list of author information is available at the end of the article
Several lines of evidence suggest that the $\alpha 7$ subtype of the nicotinic acetylcholine receptors ( $\alpha 7 \mathrm{nAChRs})$ plays an important role in the mechanism of auditory P50 gating deficits of schizophrenia, and that $\alpha 7 \mathrm{nAChR}$ agonists are potential therapeutic drugs for the deficient inhibitory processing of the P50 auditory evoked potential that contributes to the cognitive deficits in schizophrenia [717]. Tropisetron (Nabovan), a potent serotonin-3 (5hydroxytryptamine; $5-\mathrm{HT}_{3}$ ) receptor antagonist, is widely used in the treatment of patients with chemotherapyinduced or postoperative nausea and vomiting [18]. It has also been reported that tropisetron is a partial agonist at $\alpha 7 \mathrm{nAChRs}$ with a high affinity ( $\mathrm{Ki}=6.9 \mathrm{nM}$ for $\alpha 7$ nAChRs; $\mathrm{Ki}=5.3 \mathrm{nM}$ for $5-\mathrm{HT}_{3}$ receptors) [19]. We previously reported that tropisetron normalises deficient 
auditory gating in DBA/2 mice and that this effect of tropisetron was blocked by coadministration of the selective $\alpha 7 \mathrm{nAChR}$ antagonist methyllycaconitine (MLA) [20]. Furthermore, we reported that tropisetron or the selective $\alpha 7 \mathrm{nAChR}$ agonist SSR180711, but not the selective $5-\mathrm{HT}_{3}$ receptor antagonist ondansetron, could ameliorate phencyclidine (PCP)-induced cognitive deficits in mice, and that the effects of tropisetron or SSR180711 were blocked by coadministration of MLA [21,22]. In addition, we reported that a single oral administration of tropisetron $(10 \mathrm{mg}$ ) could improve deficits in auditory P50 suppression in Japanese schizophrenic patients [23]. The neuropharmacological and neurobiological effects of tropisetron demonstrated by our group suggest the need for future studies to assess the effects of tropisetron on cognitive dysfunction in schizophrenia, because P50 deficits have been previously associated with attentional deficits in schizophrenia [24]. These attentional deficits are in turn associated with psychosocial deficits that are primarily responsible the poor long-term outcome of schizophrenia [1].

This randomised, double-blind, placebo-controlled study was undertaken to examine whether adjunctive tropisetron could improve cognitive deficits and other clinical variables in patients with schizophrenia. A dose of 10 mg tropisetron was chosen since this dose has been proven effective in deficits in auditory P50 suppression of schizophrenia [23]. Risperidone was used as an antipsychotic drug because risperidone does not exhibit significant $\alpha 7 \mathrm{nAChR}$ agonism or $5-\mathrm{HT}_{3}$ receptor antagonism.

\section{Methods}

\section{Participants}

The subjects were 40 patients (19 males and 21 females; age: $35.1 \pm 7.63$ years (mean \pm SD); age range: 21 to 48 years) with schizophrenia meeting the Diagnostic and Statistical Manual of Mental Disorders, fourth edition (text revision) (DSM-IV TR) criteria [25] who were outpatients of Chiba University Hospital, Chiba, Japan (Table 1). As required by the selection criteria, they were clinically stable outpatients with no medical or neurological illness, or alcohol or other substance dependence. To be eligible for the trial, all patients had to have been taking the atypical antipsychotic drug risperidone (2 to $6 \mathrm{mg} /$ day) for at least 8 weeks, and the dose of this antipsychotic was not changed during the trial. Concomitant psychiatric medications were permissible (antidepressants, mood stabilisers, anticholinergics, and others) (Table 2), provided that patients were receiving stable doses of all of these medications in the 4 weeks preceding the trial and throughout the entire duration of the study. In all, 10 patients in the placebo group and 11 patients in the tropisetron group had taken anticholinergics (biperiden or trihexyphenidyl hydrochloride) during the trial.
The difference between the two groups in mean dosage of anticholinergics was not statistically significant (unpaired Student $\mathrm{t}$ test; $P=0.51$ ). Furthermore, 8 patients in the placebo group and 12 participants in the tropisetron group had taken benzodiazepines during the trial. However, five patients in the placebo group and three patients in the tropisetron group had taken benzodiazepines during the day. Moreover, one patient had taken lithium, and another patient had taken milnacipran and trazodone in the placebo group. One patient had taken valproic acid, and another patient had taken carbamazepine in the tropisetron group. None of the patients had taken tricyclic antidepressants. None of the patients (except dropouts) altered his or her medications during the trial. For polypharmacy, four patients in the placebo group and six patients in the tropisetron group had taken two or more kinds of drugs within anticholinergics, benzodiazepine during the day, antidepressants, or mood stabilisers. Any change in psychiatric medications at any point during the study rendered a patient ineligible to continue participation.

\section{Ethics}

The trial was approved by the Institutional Review Board of Chiba University Hospital (Chiba, Japan), and registered on the Clinical Trials Registry of the University hospital Medical Information Network (UMIN, Tokyo, Japan). All subjects provided written informed consent for their participation in the study after the procedure had been fully explained to them.

\section{Study design}

This double-blind, placebo-controlled trial used a randomisation procedure established by UMIN (UMIN 000003084). A total of 20 subjects received $10 \mathrm{mg} /$ day tropisetron (Nabovan; Novartis Pharma KK, Tokyo, Japan), and 20 subjects received a matching placebo capsule. Medications were dispensed in blister packs by the Department of Pharmacy of Chiba University Hospital.

\section{Clinical variables and cognition}

The Positive and Negative Syndrome Scale (PANSS) was used to quantify the severity of psychotic symptoms [26]. Drug-induced extrapyramidal symptoms were assessed using the Drug-Induced Extrapyramidal Symptoms Scale (DIEPSS) [27]. Patients were closely monitored for any adverse events or clinical deterioration. Quality of Life Scale (QLS) [28] scores were also measured. PANSS, DIEPSS and QLS scores were measured twice (at baseline and 8 weeks).

Cognitive function in patients with schizophrenia was measured by the Cambridge Neuropsychological Test Automated Battery (CANTAB), which consists of a series of interrelated computerised non-verbal tests of memory, attention, and executive function [29-31]. The CANTAB 
Table 1: Characteristics of subjects

\begin{tabular}{|c|c|c|c|}
\hline & Placebo group & Tropisetron group & $P$ value \\
\hline $\operatorname{Sex}(M / F)$ & $10 / 10$ & $9 / 11$ & NSa \\
\hline Age & $35.15 \pm 8.54$ & $34.96 \pm 6.82$ & NSb \\
\hline \multirow[t]{5}{*}{ Subtype } & Disorganised 1 & Disorganised 0 & NSa \\
\hline & Catatonia 1 & Catatonia 0 & \\
\hline & Paranoid 13 & Paranoid 11 & \\
\hline & Undifferentiated 2 & Undifferentiated 3 & \\
\hline & Residual 3 & Residual 6 & \\
\hline Duration of illness & $9.79 \pm 6.43$ & $12 \pm 8.67$ & NS b \\
\hline Dose of risperidone & $3.8 \pm 1.58$ & $4.03 \pm 1.59$ & NSb \\
\hline No. smoking & $5(25 \%)$ & $6(30 \%)$ & $\mathrm{NS}^{\mathrm{a}}$ \\
\hline Full IQ & $87.00 \pm 16.80$ & $87.68 \pm 18.86$ & NSb \\
\hline \multirow[t]{4}{*}{ PANSS score } & Positive: $11.8 \pm 3.12$ & Positive: $11.85 \pm 3.22$ & NSb \\
\hline & Negative: $18.45 \pm 6.66$ & Negative: $18.95 \pm 5.23$ & \\
\hline & General: $34.3 \pm 7.53$ & General: $33.75 \pm 8.69$ & \\
\hline & Total: $64.55 \pm 15.17$ & Total: $64.55 \pm 15.65$ & \\
\hline QLS total score & $77.05 \pm 15.8$ & $72.2 \pm 13.95$ & NSb \\
\hline Rate of completion & $17(85 \%)$ & $16(80 \%)$ & $\mathrm{NS}^{\mathrm{a}}$ \\
\hline \multirow[t]{3}{*}{ Reason for dropout } & Physical illness: 1 & Non-adherence: 2 & \\
\hline & Worsening of illness: 1 & Unknown: 1 & \\
\hline & $\begin{array}{l}\text { Adverse effects: } 1 \\
\text { (extrapyramidal signs) }\end{array}$ & Adverse effect: 1 (chest pain) & \\
\hline
\end{tabular}

tests used in this study were the pattern recognition memory (PRM: recognition memory for patterns), spatial recognition memory (SRM: recognition memory for spatial locations), delayed matching to sample (DMS: simultaneous and delayed perceptual matching), span length of spatial span (SSP: working memory capacity), spatial working memory (SWM: working visuospatial memory and strategy use), stocking of Cambridge (SOC: spatial planning and motor control), intra-extra dimensional set shifting (IED: rule acquisition and attentional set shifting), and rapid visual information processing (RVP; sustained visual attention) [31] tests. In this study, measurement by CANTAB was performed at baseline and 8 weeks.

\section{Auditory sensory gating P50}

Subjects were seated in a comfortable recliner and instructed to relax with their eyes open and to focus on a fixation point. The testing took place in a quiet, lighted room. The subject was monitored visually and by electroencephalogram (EEG) for signs of sleep or slow wave activity, which prompted the experimenter to speak briefly with the subject. The 120 pairs of auditory clicks were presented at a rate of 1 pair every $10 \mathrm{~s}$, with a 500-

Table 2: Other medications used by patients

\begin{tabular}{lll}
\hline Drugs & Placebo group $(\mathbf{N}=\mathbf{2 0})$ & Tropisetron group (N= 20) \\
\hline Anticholinergics & $\mathrm{N}=10$, (mean $2.2 \mathrm{mg} /$ day) & $\mathrm{N}=11,(\mathrm{mean} 1.9 \mathrm{mg} / \mathrm{day})$ \\
Benzodiazepines & $\mathrm{N}=8$, (with 5 during the day) & $\mathrm{N}=12,($ with 3 during the day) \\
Other medications & Lithium $(\mathrm{N}=1)$, milnacipran and trazodone $(\mathrm{N}=1)$ & Valproic acid ( $\mathrm{N}=1)$, carbamazepine $(\mathrm{N}=1)$ \\
\hline
\end{tabular}


ms interclick interval. The stimuli were $1 \mathrm{~ms}$ square waves amplified to $70 \mathrm{~dB}$ SPL. Recordings were performed with gold disc electrodes at $\mathrm{Fz}, \mathrm{Cz}$, and $\mathrm{Pz}$ with a forehead ground and linked earlobe reference. Data from the $\mathrm{Cz}$ site are presented, since recordings at $\mathrm{Cz}$ best discriminate schizophrenia patients from normal subjects [32]. Eye movements and blinks were monitored by electro-oculographic (EOG) recording. The resistance of all electrodes was less than $10 \mathrm{kohm}$. EEG activity was recorded by an MEB2208 8-channel system (Nihon Kohden, Tokyo, Japan) with filters at 0.5 and $100 \mathrm{~Hz}$. Data were acquired at a $500 \mathrm{~Hz}$ digitisation rate, and individual trials were stored to disk for analysis. Individual trials were rejected if the EEG or EOG voltage was greater than $\pm 70 \mu \mathrm{V}$, which is generally indicative of excessive muscle activity, eye movements, or other artefacts. The conditioning P50 wave was identified by a rater blind to the subject identity and the treatment conditions as the most positive peak between 40 and $90 \mathrm{~ms}$ after the conditioning stimulus. The test P50 wave was identified as the positive peak after the test stimulus that was closest in latency to the conditioning P50. The amplitude was defined as the difference between the positive peak and the preceding negative trough for both waves. The P50 T/ $\mathrm{C}$ ratio was calculated by dividing the test P50 amplitude by the conditioning P50 amplitude. Auditory sensory gating P50 was measured twice in all patients (at baseline and 8 weeks). Due to EEG noise, we could not measure the P50 in three patients of the placebo group.

\section{Statistical analysis}

The data were expressed as the mean \pm SD. Data analysis was performed using PASW Statistics 18.0 (formerly SPSS statistics; SPSS, Tokyo, Japan). The differences between groups were evaluated by $\mathrm{X}^{2}$ test and Student $\mathrm{t}$ test. The data on P50 and QLS in each group were assessed by paired Student $t$ test. Values of $P<0.05$ were considered to indicate statistical significance in these analyses. First, the data on the eight domains of CANTAB were assessed by repeated two-way analysis of variance (ANOVA). Next, the data of CANTAB in each group were assessed by paired Student $t$ test since there was no significant effect. We used a Bonferroni correction for multiple comparisons of CANTAB data.

\section{Trial registration}

University hospital Medical Information Network (UMIN) Clinical Trials registry No.: UMIN 000003084 http://www.umin.ac.jp.

\section{Results}

\section{Participants}

Table 1 shows the characteristics of all participants. There were no differences on their distribution in sex, age, diagnosis subtype, duration of illness, dosage of risperidone, full IQ, severity of symptoms measured with PANSS, or QLS total score (Table 1). In all, 33 participants completed the trial. Four patients in the tropisetron group dropped out due to non-adherence $(n=2)$ and adverse effects (chest pain $\mathrm{n}=1$, unknown $\mathrm{n}=1$ ); and three patients in the placebo group dropped out due to physical illness (influenza $n=1)$, worsening of illness $(\mathrm{n}=$ 1 ), and adverse effects (extrapyramidal side effects $n=1$ ) (Table 1).

\section{Adverse events}

There were no significant effects of drug treatment on vital signs, ECG, or the results of haematology measurements or serum chemistry tests. One patient in the tropisetron group complained of moderate constipation and needed to take a laxative. One female patient in the tropisetron group complained of mild chest pain, although no changes were observed in her ECG (Table 1). After conferring with the investigator she decided to discontinue the trial. Shortly after discontinuation her symptoms vanished without any medical intervention or worsening of her mental or physical status. None of the patients showed any remarkable change in their DIEPSS severity score throughout the trial, and no other adverse events were observed. Tropisetron was thus well tolerated in this trial.

\section{Auditory sensory gating P50 deficits}

Administration of tropisetron $(\mathrm{n}=16,10 \mathrm{mg} /$ day for 8 weeks; $\mathrm{t}=3.24$, degrees of freedom $(\mathrm{df})=15, P=0.006)$, but not placebo $(\mathrm{n}=14, \mathrm{t}=0.570, \mathrm{df}=13, P=0.578)$, significantly improved auditory sensory gating P50 deficits in patients with schizophrenia (Additional file 1). Next we analysed the data for non-smokers, since it is well known that smoking can affect the P50 suppression ratio and cognition in schizophrenia [33-35]. Administration of tropisetron $(\mathrm{n}=12,10 \mathrm{mg} /$ day for 8 weeks; $\mathrm{t}=2.70, \mathrm{df}=$ $11, P=0.021)$, but not placebo $(\mathrm{n}=10, \mathrm{t}=1.66, \mathrm{df}=9, P=$ $0.132)$, significantly improved auditory sensory gating P50 deficits in non-smoking patients with schizophrenia (Figure 1). Subsequently, we analysed the data of nonsmoking patients with schizophrenia.

\section{Cognitive deficits}

Repeated two-way ANOVA showed that performance on the eight domains of the CANTAB did not differ between tropisetron group and placebo group. Therefore we performed a secondary analysis using a paired Student t test in each group of non-smoking patients. Some subtests of CANTAB were significantly improved after the treatment. In the non-smoking placebo group $(n=12)$, the scores of correct rate in all trials of DMS $(\mathrm{t}=-3.94, \mathrm{df}=$ $11, P=0.002), \operatorname{SSP}(\mathrm{t}=-2.60, \mathrm{df}=11, P=0.025)$, and a 


\section{Tropisetron Group (N=12)}

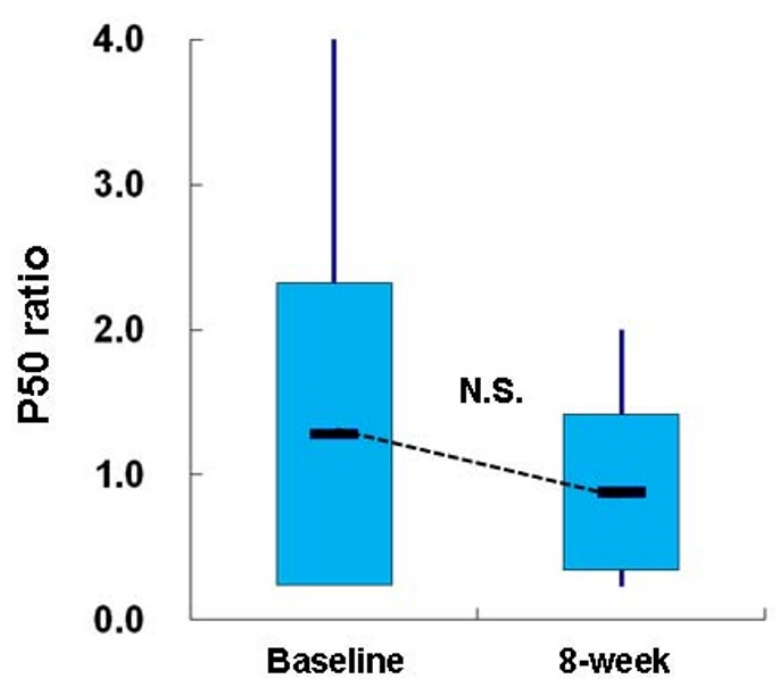

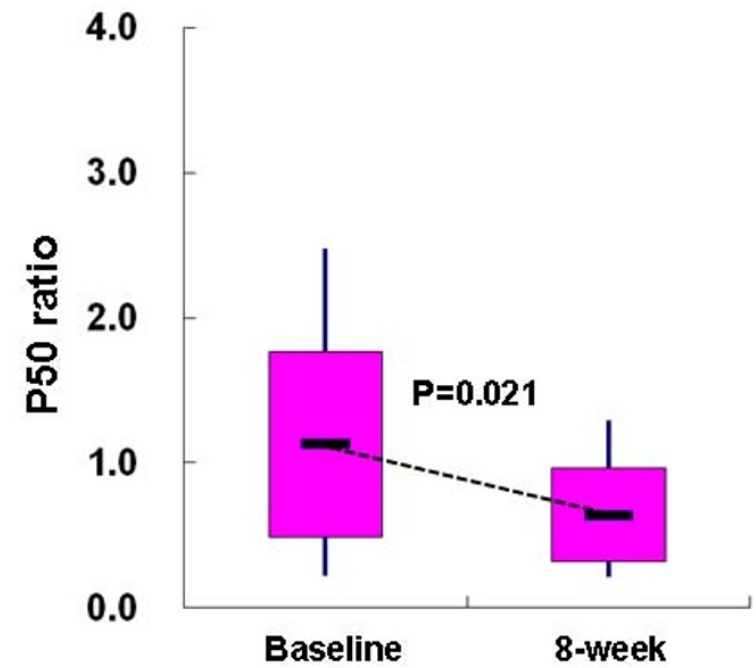

Figure 1 Effect of tropisetron on auditory sensory gating P50 deficits in non-smoking patients with schizophrenia. The ratio of test P50 amplitude to conditioning amplitude was measured at baseline and 8 weeks after placebo or tropisetron treatment. Tropisetron $(t=2.70$, degrees of freedom $(\mathrm{d} f)=11, P=0.021)$, but not placebo $(t=1.66, \mathrm{df}=9, P=0.132)$, significantly decreased the $P 50$ ratio in non-smoking patients with schizophrenia. Data are from the placebo group $(n=10)$ and the tropisetron group $(n=12)$.

prime of $\operatorname{RVP}(\mathrm{t}=-2.77, \mathrm{df}=11, P=0.018)$ were improved after placebo treatment, but SSP and RVP were not significant after a Bonferroni correction. However, DMS was still significant after a Bonferroni correction, suggesting that a practice effect was operative. Furthermore, the scores for RVP were significantly improved after treatment with tropisetron $(\mathrm{t}=-5.78, \mathrm{df}=11, P<$ 0.001); which was still significant after a Bonferroni correction (Figure 2). Other subtests of CANTAB were not changed by this trial. In addition, we did not observe any significant correlation between P50 changes and RVP changes in the non-smoking patients with schizophrenia.

\section{Psychotic symptoms and QLS}

Total and subscale scores (positive symptoms, negative symptoms, or general psychopathological symptoms) of PANSS were not altered by treatment with tropisetron or placebo (Additional file 2). Furthermore, administration of tropisetron significantly $(\mathrm{t}=-2.42, \mathrm{df}=15, P=0.029)$ increased QLS total scores in total patients $(n=16)$ with schizophrenia, whereas administration of placebo did not change QLS total scores $(\mathrm{t}=-0.72, \mathrm{df}=13, P=0.487$ ) (Additional file 3). However, administration of tropisetron did not alter QLS total scores in non-smoking patients $(n=12)$. In addition, we did not observe any significant correlation between P50 changes and QLS score changes in the total or non-smoking patients with schizophrenia.

\section{Effects of tropisetron on smoking patients}

Moreover, we did not observe any effect of tropisetron on the P50 ratio, psychotic symptoms, QLS, or cognitive functions in the smoking patients (tropisetron group $\mathrm{n}=$ 4; placebo group $n=5$ ), although the sample number in each group was too small to reach any definitive conclusions in regard to these parameters. In addition, tropisetron did not affect smoking status (for example, the number of cigarettes) in the smoking patients $(n=4)$.

\section{Discussion}

The major findings of this double-blind, placebo-controlled study are that administration of tropisetron, but not placebo, significantly improved auditory sensory gating P50 deficits in patients with schizophrenia, and that tropisetron had a significant impact on the sustained visual attention measured with the RVP subtest of CAN$\mathrm{TAB}$ in non-smoking patients. Previously, we reported that the effect of a single administration of tropisetron (10 mg) on P50 deficits in patients with schizophrenia was significant only for the non-smokers [23], indicating that smoking status can affect P50 deficits in schizophrenia. In the present study, we also found that administration of tropisetron (10 mg/day for 8 weeks), but not placebo, significantly improved P50 deficits in non-smoking patients with schizophrenia, suggesting that chronic as well as acute administration of tropisetron $(10 \mathrm{mg})$ can improve P50 deficits in non-smoking patients with 


\section{Placebo Group ( $\mathrm{N}=12)$}

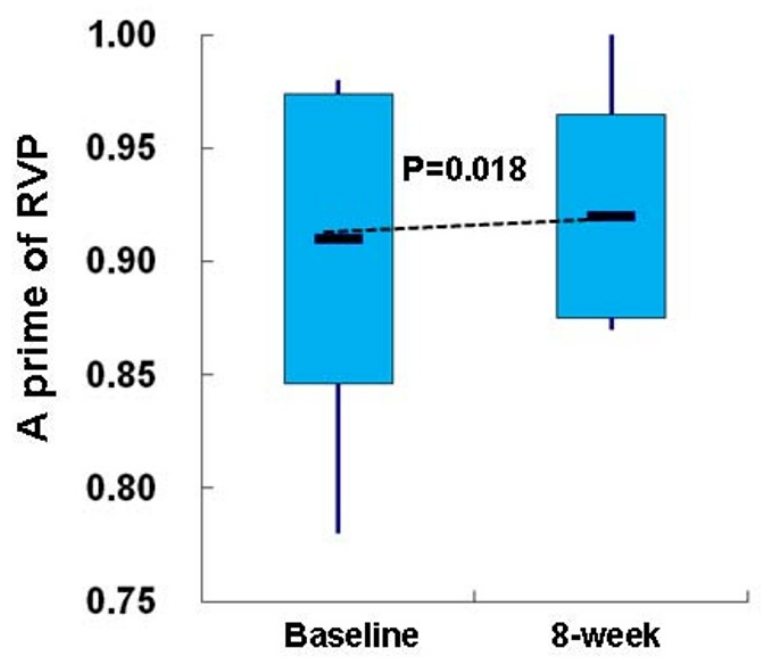

\section{Tropisetron Group ( $\mathrm{N}=12)$}

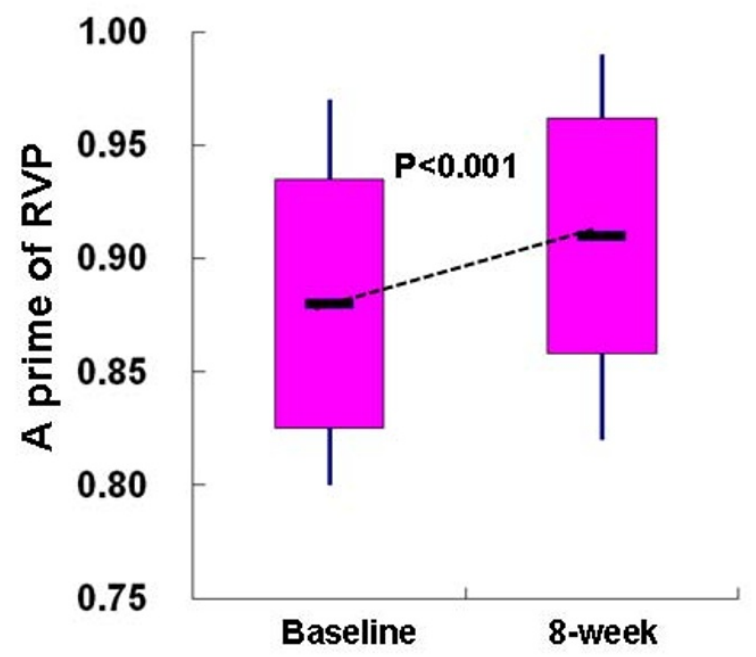

Figure 2 Rapid visual information processing (RVP) scores on Cambridge Neuropsychological Test Automated Battery (CANTAB) subtests for non-smoking patients with schizophrenia during treatment placebo and tropisetron. There were significantly different $s c 0 r e s$ for RVP ( $t=-$ 5.78 , degrees of freedom $(\mathrm{d} f)=11, P<0.001)$ in the tropisetron group, but not the placebo group. Data are from the placebo group $(n=12)$ and the tropisetron group $(n=12)$.

schizophrenia. Interestingly, we found that sustained visual attention as measured with the RVP subtest of CANTAB in non-smoking patients was significantly improved by tropisetron treatment, but not by placebo treatment. It has been reported that the $\alpha 7 \mathrm{nAChR}$ agonist 3-(2,4-dimethoxybenzylidene) anabaseine (DMXBA) had effects on auditory P50 deficits, attention/vigilance and working memory Measurement and Treatment Research to Improve Cognition in Schizophrenia (MATRICS) [36,37] domains in stable and non-smoking patients with schizophrenia [13,14]. Auditory sensory P50 deficits have also been shown to be associated with attentional deficits in schizophrenia [24]. Unexpectedly, we did not observe any significant correlation between P50 changes and RVP subtest changes in the non-smoking patients because of the small sample size. A further study using a large sample size will be necessary. Taken together, it is likely that the improvement of P50 deficits by tropisetron is involved in the beneficial effects of this drug on attention. Furthermore, the results of this trial suggest that stimulation at $\alpha 7 \mathrm{nAChRs}$ by $\alpha 7 \mathrm{nAChR}$ agonists can lead to improvement in auditory P50 deficits and aspects of cognitive performance such as attention.

Several studies strongly suggest that cognitive deficits have a major impact on QOL in patients with schizophrenia $[1,4,38,39]$. A recent review of longitudinal studies demonstrated that cognition deficits are associated with functional outcomes in schizophrenia, and that cognitive assessment predicts later functional outcomes in patients, suggesting a rationale for psychopharmacological interventions for cognitive deficits [4]. It is thus significant that, in the present study, an 8-week treatment with tropisetron could improve the QOL in total patients with schizophrenia although this is not significant in the nonsmoking patients. In addition, we did not observe any significant correlation between P50 changes and QLS score change in the non-smoking patients because of small sample size. A further study using a large sample size will be necessary. Freedman et al. [14] reported that treatment with DMXB-A significantly improved two subscales (for example, alogia and anhedonia) of the Scale for the Assessment of Negative Symptoms (SANS), but not the total score of the Brief Psychiatric Rating Scale (BPRS). The effects of DMXB-A on negative symptoms are also noteworthy, as these negative symptoms are generally resistant to antipsychotic drugs. However, in this trial, we did not observe any effect of tropisetron on the positive symptoms or negative symptoms scores of PANSS. Further detailed studies regarding the effects of tropisetron on psychotic symptoms such as negative symptoms will therefore be necessary.

Tropisetron is also a potent antagonist at $5-\mathrm{HT}_{3}$ receptors. At present, it is unclear whether improvement of P50 deficits by tropisetron is mediated via direct agonist effects on $\alpha 7 \mathrm{nAChRs}$ or via direct antagonist effects on $5-\mathrm{HT}_{3}$ receptors. Previously, we reported that tropise- 
tron, but not the selective $5-\mathrm{HT}_{3}$ receptor antagonist ondansetron, attenuated PCP-induced cognitive deficits in mice, and that this effect of tropisetron was blocked by coadministration of the selective $\alpha 7 \mathrm{nAChR}$ antagonist MLA [21]. These results suggest that activation of $\alpha 7$ nicotinic receptors by tropisetron is likely to play a role in the mechanism of action of tropisetron [17,21]. In contrast, the selective $5-\mathrm{HT}_{3}$ receptor antagonist ondansetron was reported to improve deficient auditory gating in DBA/2 mice [40]. In addition, ondansetron was shown to be effective in auditory P50 deficits, negative symptoms and cognitive symptoms in patients with schizophrenia [41-44]. These results suggest that $5-\mathrm{HT}_{3}$ receptor antagonism may contribute to the action of tropisetron. Therefore, a clinical/feasibility study comparing tropisetron versus ondansetron with or without placebo will help to determine whether the addition of the $\alpha 7 \mathrm{nAChR}$ partial agonism of tropisetron has enhanced effects versus simply adding a $5-\mathrm{HT}_{3}$ receptor antagonist. In contrast, it seems that DMXB-A addition to an atypical antipsychotic or versus placebo has the advantage of specificity of action. Nonetheless, in order to confirm the role of $\alpha 7$ nAChRs in the treatment of schizophrenia, a randomised double-blind, placebo-controlled study of the selective $\alpha 7$ $n A C h R$ agonists in patients with schizophrenia would be necessary.

There were no adverse side effects associated with the tropisetron trial. Tropisetron, which is already approved for human use outside the USA, is widely used in the treatment of patients with chemotherapy-induced or postoperative nausea and vomiting [18]. Thus, it was not surprising that tropisetron $(10 \mathrm{mg})$ was well tolerated in this trial. Freedman et al. [14] reported that nausea occurred in $45 \%$ of a group of patients receiving a high dose of the $\alpha 7$ nAChR agonist DMXB-A, and this was suggested to be due to the known effects of nicotinic agonists on gastrointestinal mobility. Considering the high incidence of nausea among patients treated with $\alpha 7$ nAChR agonists (for example, DMXB-A), it is likely that $\alpha 7 \mathrm{nAChR}$ agonists (for example, tropisetron) with $5-\mathrm{HT}_{3}$ receptor antagonism will be suitable therapeutic drugs for schizophrenia, since $5-\mathrm{HT}_{3}$ receptor antagonists are therapeutic drugs for nausea [45].

It has been reported that varenicline, a partial agonist at $\alpha 4 \beta 2 \mathrm{nAChRs}$ as well as a full agonist at $\alpha 7 \mathrm{nAChR}$ agonists, was not effective in the auditory P50 deficits in patients with schizophrenia [46]. The precise reasons underlying the lack of varenicline on P50 deficits is currently unknown. One possibility is that the receptor desensitisation may occur by the time measurement because varenicline is a full agonist at the $\alpha 7 \mathrm{nAChRs.} \mathrm{A}$ further detailed study using a selective partial agonist and a selective full agonist will be necessary. Another possibil- ity is that there was incomplete absorption of a single dose of varenicline, leading to insufficient levels of the drug in the brain [46]. Furthermore, Freedman [47] reported the case of a patient with schizophrenia who received varenicline and experienced an activated psychotic relapse. The US Food and Drug Administration (FDA) and the Institute for Safe Medication Practices stated that serious neuropsychiatric symptoms including changes in behaviour, agitation, depressed mood, suicidal ideation and attempted and completed suicide have occurred in patients with taking varenicline [48]. Therefore, close monitoring of patients prescribed this drug will be warranted. In contrast, tropisetron and DMXB-A have not been reported to induce psychosis in patients with schizophrenia (this study, and [14]). Therefore, it is likely that tropisetron and DMXB-A have lower risk of inducing psychosis than varenicline although the reasons underlying this difference are currently unknown.

Inhibitory interneurons with $\alpha 7 \mathrm{nAChRs}$ are possible candidates for medication to ameliorate the habituation of auditory responses in the hippocampus, because activation of the interneurons via $\alpha 7 \mathrm{nAChRs}$ would increase the inhibitory synaptic input to pyramidal neurons and thereby diminish the responsiveness of these pyramidal neurons to sensory stimulation [49]. This parallels a study of postmortem human tissue that documented a decreased expression of hippocampal $\alpha 7$ nAChRs in schizophrenic patients [49]. Furthermore, it has been reported that $\left[{ }^{125} \mathrm{I}\right] \alpha$-bungarotoxin binding to $\alpha 7 \mathrm{nAChRs}$ is reduced in the thalamic reticular nucleus of schizophrenic subjects [50], and that $\alpha 7 \mathrm{nAChR}$ protein levels are reduced in the frontal cortex in patients with schizophrenia [51]. Thus, it seems that schizophrenic patients have fewer $\alpha 7 \mathrm{nAChRs}$ in the hippocampus, a condition which may lead to failure of cholinergic activation of inhibitory interneurons, clinically manifested as decreased gating of the response to sensory stimulation [17]. Therefore, it is of great interest to study whether the density of $\alpha 7$ nAChRs is altered in the intact brain of patients with schizophrenia. A positron emission tomography (PET) study using the selective $\alpha 7 \mathrm{nAChR}$ ligand $\left.{ }^{11} \mathrm{C}\right]$ CHIBA-1001 $[52,53]$ in the intact brains of patients with schizophrenia is currently underway.

Finally, several limitations of this study should be mentioned. One of the main limitations of this trial was its small sample size (total $n=40$ ) and the use of only one dose $(10 \mathrm{mg})$ of tropisetron. The dose $(10 \mathrm{mg})$ of tropisetron used in this study was well tolerated and resulted in significant effects on P50 deficits, and aspects of cognitive performance such as attention. However, it is currently unclear if other dosing approaches would be more efficacious. Very recently, using $\left[{ }^{11} \mathrm{C}\right] \mathrm{CHIBA}-1001$ and PET, we found that a single oral administration of tropisetron $(5,10$ or $20 \mathrm{mg})$, but not ondansetron, could bind to 
$\alpha 7$ nAChRs in the intact human brain in a dose-dependent manner (Ishikawa M, Ishii K, Wu J, Toyohara J, Sakata M, Oda K, Kimura Y, Iyo M, Ishiwata K, Hashimoto K. unpublished results). Therefore, a randomised double-blind, placebo-controlled study of higher doses (for example, $20 \mathrm{mg}$ ) of tropisetron in patients with schizophrenia using a larger sample will be needed. In addition, the duration of treatment in this trial (8 weeks) was fairly short. A double-blind, placebo-controlled study with a longer duration (for example, 48 weeks) of tropisetron treatment would also be of use. Another limitation was the possible presence of practice effects for CANTAB [31], which may have been responsible for the improvement of cognitive performance in the placebo group of this study. In this study, sustained visual attention measured with the RVP subtest was significantly improved by tropisetron, but not by placebo, in non-smokers, suggesting that the effects of tropisetron on RVP might not be due to practice effects. Furthermore, Freedman et al. [14] also observed practice effects in a study using the MATRICS Consensus Cognitive Battery to investigate the efficacy of DMXB-A. Therefore, further detailed studies to validate the use of other cognitive batteries in patients taking tropisetron, such as MATRICS, Brief Assessment of Cognition in Schizophrenia (BACS) [54], and the CogState Schizophrenia Battery [55], would be of interest.

In conclusion, the results of the present feasibility study investigating adjunctive tropisetron as a treatment strategy for cognitive deficits in schizophrenia are promising. Similar to ondansetron and DMXB-A, tropisetron was well tolerated in this trial, and was associated with no untoward effects. If these initial pilot findings are confirmed in larger randomised controlled trials, tropisetron will be a potential therapeutic drug for the treatment of cognitive deficits and QOL in patients with schizophrenia.

\section{Additional material}

Additional file 1 Effect of tropisetron on auditory sensory gating P50 deficits in all patients with schizophrenia. The ratio of test P50 amplitude to conditioning amplitude was measured at baseline and 8 weeks after placebo or tropisetron treatment. Tropisetron, but not placebo, significantly decreased the P50 ratio in patients with schizophrenia. Data are the mean of the placebo group $(n=14)$ and the tropisetron group $(n=16)$.

Additional file $\mathbf{2}$ Supplemental table. Scores for Positive and Negative Syndrome Scale (PANSS) in patients with schizophrenia.

Additional file 3 Supplemental table. Changes of Quality of Life Scale (QLS) scores in patients with schizophrenia.

\section{Competing interests}

$\mathrm{KH}$ and $\mathrm{Ml}$ have a patent for 'the use of tropisetron in neuropsychiatric diseases' through Chiba University. All authors report no competing interests related with this study.

\section{Authors' contributions}

AS, YS, TN, THash, TY, THase, THar, NK, TS, MF, GF, MN, and Ml recruited the patients enrolled in this study. AS, YS, MI and $\mathrm{KH}$ conducted the statistical anal- ysis. AS and $\mathrm{KH}$ wrote the manuscript. $\mathrm{KH}$ is the principal investigator of this study. All authors read and approved the final manuscript.

\section{Acknowledgements}

This study was supported by grants from the Stanley Research Foundation (to KH, ID no. 05T-700) and the Program for the Promotion of Fundamental Studies in Health Sciences of the National Institute of Biomedical Innovation, Japan (to KH, ID no. 06-46). The authors thank Daisuke Matsuzawa (Chiba University), Tomoko Watanabe (Hamamatsu University), and Professor Yoshio Minabe (Kanazawa University) for their valuable advice on the use of CANTAB. The authors are very grateful to Tsuyoshi Sasaki (Chiba University), Daiji Sakurai (Kisarazu Hospital), Hiroshi Yamanaka and Makoto Asano (Chiba Psychiatric Medical Center) for recruiting the patients. The authors thank Professor Nori Takei (Hamamatsu University) for his advice. The authors also thank the staff of the Department of Pharmacy, Chiba University Hospital and Chiba University Hospital Clinical Research Center for their assistance with this trial.

\section{Author Details}

1Department of Psychiatry, Chiba University Hospital, Chiba, Japan, 2Department of Mental Health, Teikyo University Chiba Medical Center, Chiba, Japan, ${ }^{3}$ Department of Psychiatry, Chiba University Graduate School of Medicine, Chiba, Japan and ${ }^{4}$ Division of Clinical Neuroscience, Chiba University Center for Forensic Mental Health, Chiba, Japan

Received: 1 April 2010 Accepted: 24 June 2010

Published: 24 June 2010

\section{References}

1. Green M: What are the functional consequences of neurocognitive deficits in schizophrenia? Am J Psychiatry 1996, 153:321-330.

2. Harvey PD, Keefe RS: Studies of cognitive change in patients with schizophrenia following novel antipsychotic treatment. Am J Psychiatry 2001, 158:176-184.

3. Green MF, Nuechterlein KH, Gold JM, Barch DM, Cohen J, Essock S, Fenton WS, Frese F, Goldberg TE, Heaton RK, Keefe RS, Kern RS, Kraemer H, Stover E, Weinberger DR, Zalcman S, Marder SR: Approaching a consensus cognitive battery for clinical trials in schizophrenia: the NIMH-MATRICS conference to select cognitive domains and test criteria. Biol Psychiatry 2004, 56:301-307.

4. Green MF, Kern RS, Heaton RK: Longitudinal studies of cognition and functional outcome in schizophrenia: implications for MATRICS. Schizophrenia Res 2004, 72:41-51.

5. Harvey PD, Helldin L, Bowie CR, Heaton RK, Olsson AK, Hjärthag F, Norlander T, Patterson TL: Performance-based measurement of functional disability in schizophrenia: a cross-national study in the United States and Sweden. Am J Psychiatry 2009, 166:821-827.

6. Meltzer HY, McGurk SR: The effects of clozapine, risperidone, and olanzapine on cognitive function in schizophrenia. Schizophrenia Bull 1999, 25:233-255

7. Freedman R, Adler LE, Bickford P, Byerley W, Coon H, Cullum CM, Griffith J, Harris JG, Leonard S, Miller C, Myles-Worsey M, Nagamoto HT, Rose G, Waldo M: Schizophrenia and nicotinic receptors. Harv Rev Psychiatry 1994, 2:179-192.

8. Freedman R, Coon H, Myles-Worsley M, Orr-Urtreger A, Olincy A, Davis A, Polymeropoulos M, Holik J, Hopkins J, Hoff M, Rosenthal J, Waldo MC, Reimherr F, Wender P, Yaw J, Young DA, Breese CR, Adams C, Patterson D, Adler LE, Kruglyak L, Leonard S, Byerley W: Linkage of a neurophysiological deficit in schizophrenia to a chromosome 15 locus. Proc Natl Acad Sci USA 1997, 94:587-592.

9. Leonard S, Gault J, Hopkins J, Logel J, Vianzon R, Short M, Drebing C, Berger R, Venn D, Sirota P, Zerbe G, Olincy A, Ross RG, Adler LE, Freedman $\mathrm{R}$ : Association of promoter variants in the a7 nicotinic acetylcholine receptor subunit gene with an inhibitory deficit found in schizophrenia. Arch Gen Psychiatry 2002, 59:1085-1096.

10. Leonard S, Freedman R: Genetics of chromosome 15q13-q14 in schizophrenia. Biol Psychiatry 2006, 60:115-122.

11. Martin LF, Kem WR, Freedman R: a7 nicotinic receptor agonists: potential new candidates for the treatment of schizophrenia. Psychopharmacology (Berlin) 2004, 174:54-64. 
12. Hashimoto K, Koike K, Shimizu E, lyo M: a7 Nicotinic receptor agonists as potential therapeutic drugs for schizophrenia. Curr Med Chem CNS Agents 2005, 5:171-184.

13. Olincy A, Harris JG, Johnson LL, Pender V, Kongs S, Allensworth D, Ellis J, Zerbe GO, Leonard S, Stevens KE, Stevens JO, Martin L, Adler LE, Soti F, Kem WR, Freedman R: Proof-of-concept trial of an a7 nicotinic agonist in schizophrenia. Arch Gen Psychiatry 2006, 63:630-638.

14. Freedman R, Olincy A, Buchanan RW, Harris JG, Gold JM, Johnson L, Allensworth D, Guzman-Bonilla A, Clement B, Ball MP, Kutnick J, Pender V, Martin LF, Stevens KE, Wagner BD, Zerbe GO, Soti F, Kem WR: Initial phase 2 trial of a nicotinic agonist in schizophrenia. Am J Psychiatry 2008, 165:1040-1047.

15. Buchanan RW, Freedman R, Javitt DC, Abi-Dargham A, Lieberman JA: Recent advances in the development of novel pharmacological agents for the treatment of cognitive impairments in schizophrenia. Schizophrenia Bull 2007, 33:1120-1130.

16. Olincy A, Stevens KE: Treating schizophrenia symptoms with an a7 nicotinic agonist, from mice to men. Biochem Pharmacol 2007 74:1192-1201

17. Toyohara J, Hashimoto K: a7 Nicotinic receptor agonists as potential therapeutic drugs for treatment of cognitive dysfunction associated with Alzheimer's disease and schizophrenia. Open Med Chem J 2010, 4:37-56.

18. Simpson K, Spencer CM, McClellan KJ: Tropisetron: an update of its use in the prevention of chemotherapy-induced nausea and vomiting. Drugs 2000, 59:1297-1315

19. Macor JE, Gurley D, Lanthorn T, Loch J, Mack RA, Mullen G, Tran O, Wright N, Gordon JC: The 5-HT3 antagonist tropisetron (ICS 205-930) is a potent and selective a7 nicotinic receptor partial agonist. Bioorg Med Chem Lett 2001, 11:319-321.

20. Hashimoto K, lyo M, Freedman R, Stevens KE: Tropisetron improves deficient inhibitory auditory processing in DBA/2 mice: role of $a 7$ nicotinic acetylcholine receptors. Psychopharmacology (Berlin) 2005, 183:13-19.

21. Hashimoto K, Fujita Y, Ishima T, Hagiwara H, Iyo M: Phencyclidineinduced cognitive deficits in mice are improved by subsequent subchronic administration of tropisetron: role of a7 nicotinic receptors. Eur J Pharmacol 2006, 553:191-195.

22. Hashimoto K, Ishima T, Fujita Y, Matsuo M, Kobashi T, Takahagi M, Tsukada $\mathrm{H}$, lyo M: Phencyclidine-induced cognitive deficits in mice are improved by subsequent subchronic administration of the novel selective a7 nicotinic receptor agonist SSR180711. Biol Psychiatry 2008, 63:92-97.

23. Koike K, Hashimoto K, Takai N, Shimizu E, Komatsu N, Watanabe H, Nakazato M, Okamura N, Stevens KE, Freedman R, lyo M: Tropisetron improves deficits in auditory P50 suppression in schizophrenia. Schizophrenia Res 2005, 76:67-72.

24. Cullum CM, Harris JG, Waldo MC, Smernoff E, Madison A, Nagamoto HT, Griffith J, Adler LE, Freedman R: Neurophysiological and neuropsychological evidence for attentional dysfunction in schizophrenia. Schizophrenia Res 1993, 10:131-141.

25. American Psychiatric Association: Diagnostic and Statistical Manual of Mental Disorders (DSM)-IV-TR Arlington, VA, USA: American Psychiatric Press; 2000

26. Kay SR, Fiszbein A, Opler LA: The positive and negative syndrome scale (PANSS) for schizophrenia Evaluation and diagnosis of drug-induced extrapyramidal symptoms: commentary on the DIEPSS and guide to its usage [in Japanese]. Schizophrenia Bull 1987, 13:261-276.

27. Inada T: Evaluation and diagnosis of drug-induced extrapyramidal symptoms: commentary on the DIEPSS and guide to its usage [in Japanese] Tokyo, Japan: Seiwa; 1996.

28. Heinrichs DW, Hanlon TE, Carpenter WT: The Quality of Life Scale: an instrument for rating the schizophrenia deficits syndrome. Schizophrenia Bull 1984, 10:388-398.

29. Sahakian BJ, Owen AM: Computerized assessment in neuropsychiatry using CANTAB: discussion paper. JR Soc Med 1992, 85:399-402.

30. Fray PJ, Robbins TW, Sahakian BJ: Neuropsychiatric applications of CANTAB. Int J Ger Psychiatry 1996, 11:329-336.

31. Levaux MN, Potvin S, Sepehry AA, Sablier J, Mendrek A, Stip E: Computerized assessment of cognition in schizophrenia: promises and pitfalls of CANTAB. Eur Psychiatry 2007, 22:104-115.
32. Clementz BA, Geyer MA, Braff DL: Poor P50 suppression among schizophrenia patients and their first-degree biological relatives. Am J Psychiatry 1998, 155:1691-1694.

33. Adler LE, Hoffer LD, Wiser A, Freedman R: Normalization of auditory physiology by cigarette smoking in schizophrenic patients. Am J Psychiatry 1993, 150:1856-1861.

34. George TP, Vessicchio JC, Termine A, Sahady D, Head CA, Pepper T, Kosten TR, Wexler BE: Effects of smoking abstinence on visuospatial working memory function in schizophrenia. Neuropsychopharmacol 2002, 26:75-85.

35. Sacco KA, Termine A, Seyal A, Dudas MM, Vessicchio JC, Krishnan-Sarin S, Jatlow PI, Wexler BE, George TP: Effects of cigarette smoking on spatial working memory and attentional deficits in schizophrenia: involvement of nicotinic receptor mechanisms. Arch Gen Psychiatry 2005, 62:649-659.

36. Nuechterlein KH, Green MF, Kern RS, Baade LE, Barch DM, Cohen JD, Essock S, Fenton WS, Frese FJ, Gold JM, Goldberg T, Heaton RK, Keefe RS, Kraemer H, Mesholam-Gately R, Seidman LJ, Stover E, Weinberger DR, Young AS, Zalcman S, Marder SR: The MATRICS Consensus Cognitive Battery, part 1: test selection, reliability, and validity. Am J Psychiatry 2008, 165:203-213.

37. Kern RS, Nuechterlein KH, Green MF, Baade LE, Fenton WS, Gold JM, Keefe RS, Mesholam-Gately R, Mintz J, Seidman L, Stover E, Marder SR: The MATRICS Consensus Cognitive Battery, part 2: co-norming and standardization. Am J Psychiatry 2008, 165:214-220.

38. Breier A, Schreiber JL, Dyer J, Pickar D: National Institute of Mental Health longitudinal study of chronic schizophrenia. Prognosis and predictors of outcome. Arch Gen Psychiatry 1991, 48:239-246.

39. Ho BC, Nopoulos P, Flaum M, Arndt S, Andreasen NC: Two-year outcome in first-episode schizophrenia: predictive value of symptoms for quality of life. Am J Psychiatry 1998, 155:1 196-1201.

40. Wildeboer KM, Zheng L, Choo KS, Stevens KE: Ondansetron results in improved auditory gating in DBA/2 mice through a cholinergic mechanism. Brain Res 2009, 1300:41-50.

41. Adler LE, Cawthra EM, Donovan KA, Harris JG, Nagamoto HT, Olincy A, Waldo MC: Improved P50 auditory gating with ondansetron in medicated schizophrenia patients. Am J Psychiatry 2005, 162:386-388.

42. Levkovitz Y, Arnest G, Mendlovic S, Treves I, Fennig S: The effect of ondansetron on memory in schizophrenic patients. Brain Res Bull 2005, 65:291-295.

43. Zhang ZJ, Kang WH, Li Q, Wang XY, Yao SM, Ma AQ: Beneficial effects of ondansetron as an adjunct to haloperidol for chronic, treatmentresistant schizophrenia: a double-blind, randomized, placebocontrolled study. Schizophr Res 2006, 88:102-110.

44. Akhondzadeh S, Mohammadi N, Noroozian M, Karamghadiri N, Ghoreishi A, Jamshidi AH, Forghani S: Added ondansetron for stable schizophrenia: a double blind, placebo controlled trial. Schizophr Res 2009, 107:206-212.

45. Hashimoto K: Nausea associated with a nicotinic agonist therapy in schizophrenia. Clin Psychopharmacol Neurosci 2009, 7:26-27.

46. Waldo MC, Woodward L, Adler LE: Varenicline and P50 auditory gating in medicated schizophrenic patients: A pilot study. Psychiatry Res 2010, 175:179-180.

47. Freedman R: Exacerbation of schizophrenia by varenicline. Am J Psychiatry 2007, 164:1269.

48. Moore TJ, Cohen MR, Furberg CD: Strong safety signal seen for new varenicline risks. Institute of Safe Medication Practices [http:// www.ismp.org/docs/vareniclinestudy.asp].

49. Freedman $R$, Hall $M$, Adler LE, Leonard S: Evidence in postmortem brain tissue for decreased numbers of hippocampal nicotinic receptors in schizophrenia. Biol Psychiatry 1995, 38:22-33.

50. Court J, Spurden D, Lloyd S, McKeith I, Ballard C, Cairns N, Kerwin R, Perry $R$, Perry E: Neuronal nicotinic receptors in dementia with Lewy bodies and schizophrenia: $\mathrm{a}$-bungarotoxin and nicotine binding in the thalamus. J Neurochem 1999, 73:1590-1597.

51. Guan ZZ, Zhang X, Blennow K, Nordberg A: Decreased protein level of nicotinic receptor a7 subunit in the frontal cortex from schizophrenic brain. NeuroReport 1999, 10:1779-1782.

52. Hashimoto K, Nishiyama S, Ohba H, Matsuo M, Kobashi T, Takahagi M, lyo M, Kitashoji T, Tsukada H: [ $\left.{ }^{11} \mathrm{C}\right] \mathrm{CHIBA}-1001$ as a novel PET ligand for a7 nicotinic receptors in the brain: a PET study in conscious monkeys. PLOS ONE 2008, 3:e3231. 
53. Toyohara J, Sakata M, Wu J, Ishikawa M, Oda K, Ishii K, Iyo M, Hashimoto K, Ishiwata K: Preclinical and the first clinical studies on $\left[{ }^{11} \mathrm{C}\right] \mathrm{CHIBA}-1001$ for mapping a7 nicotinic receptors by positron emission tomography. Ann Nucl Med 2009, 23:301-309.

54. Keefe RS, Goldberg TE, Harvey PD, Gold JM, Poe MP, Coughenour L: The Brief Assessment of Cognition in Schizophrenia: reliability, sensitivity, and comparison with a standard neurocognitive battery. Schizophrenia Res 2004, 68:283-297.

55. Pietrzak RH, Olver J, Norman T, Piskulic D, Maruff P, Snyder PJ: A comparison of the CogState Schizophrenia Battery and the Measurement and Treatment Research to Improve Cognition in Schizophrenia (MATRICS) Battery in assessing cognitive impairment in chronic schizophrenia. J Clin Exp Neuropsychol 2010, 31:848-859.

doi: $10.1186 / 1744-859 X-9-27$

Cite this article as: Shiina et al., A randomised, double-blind, placebo-controlled trial of tropisetron in patients with schizophrenia Annals of General Psychiatry 2010, 9:27

Submit your next manuscript to BioMed Central and take full advantage of:

- Convenient online submission

- Thorough peer review

- No space constraints or color figure charges

- Immediate publication on acceptance

- Inclusion in PubMed, CAS, Scopus and Google Scholar

- Research which is freely available for redistribution

Submit your manuscript at www.biomedcentral.com/submit
C) Biomed Central 